\title{
Die Zerstörung der freien Advokatur im Nationalsozialismus ${ }^{*-}$
}

\author{
1. Anwälte zwischen Widerstand und Anpassung im NS-Staat
}

Die deutsche Anwaltschaft ist wie kaum eine zweire Berufsgruppe in Deutschland Opfer nationalsozialistischen Terrors geworden.' 4400 Rechtsanwälte, d. h. mehrals ein Fünfrel der Anwaltschaft wurde mit Berulsverbor belegt, ins Exil getrieben, verschleppt oder ermordet. Die Rolle des Verteidigers wurde in ihr direktes Gegenreil verkehrt. An die Stelle des Strafverteidigers, der mir dem Veriahrensrecht, den Waffen der Revision und der Unschuldsvermutung gerüster den Angcklagten vor der Allmacht des Staates zu schützen hatte, trat der deutsche Rechtswahrer, ohne Revisions- und Berufungsmöglichkeit vor den Sondergerichten und ohne Beweisantrags- und Bewcislührungsrecht vor den Strafgerichten insgesame, abgeschnitten vom Mandancen und in Prozessen gegen politisch Mißliebige sogar auch ohne Einsicht in dic Anklageschrift. Hatte er nichr, wie beim Volksgerichrshof, gar die Rolle, Todesurteile zu beantragen, so war er bestenfalls der Fürsprecher, der das Urteil dem Angeklagten verständlich zu machen hatte und das Gnadengesuch formulierte.

Standespolicisch wurden die Srrukturen erheblich geänderc. Das Führerprinzip und die neuc Reichsrechtsanwaltskammer lösten das Kollegialprinzip ab, die Ehrengerichısbarkeit geriet in die Hände der Partei und sanktionierte z. B. die Verweigerung des ,deurschen Grußesu, staatsabrägliche Äußerungen und das Versäumen der Wahl. Aus dem deutschen Anwalesverein wurde 1933 dic Fachgruppe Rechtsanwälte im Bund Nationalsozialistischer Deurscher Juristen, später umbenannt in den NSRechtswahrerbund.

Geradc diese Uinwandlung zeigt aber auch, daß die deutsche Anwaltschaft nicht nur Erleidender, sondern auch Handelnder war, wenn man die freiwilligen Gleichschaltungsbemühungen in den einzelnen Verbänden bis hin zum Vorstand des DAV verfolgt. Verwirrender noch wird die Einschätzung bestimmter berufspolicischer Entwicklungen aus dieser Zeit. Das Rechtsberatungsmißbrauchsgeserz von 1935 ist heute noch in Kraft und von der Rechtsprechung als Schurzgesctz für den Anrwaltsstand angesehen. Ebenfalls beibehalten wurde eine Zentrale der Anwaltskammem in der Bundesrechusanwaltskammer, die damir die 1933 durch Norverordnung eingeführte Reichsrechtsanwalrskammer fortsetzt.

Der Aufsatz scellı Vorüberlegungen für ein Buch zum gleichen Thema dar. An dem Buch, auf dessen Matcrial der Aulsalz zurückgreift, arbeiten stud. jur. Raimund Körn«t. Anne v. Alven-Döríng u. a. mit. Ich biles, mir für weitere Arbeiten zum Thema. Anwaltschafu im Naxionalsocialismus" Berichte von Zeitzeugen mitzutcilen.

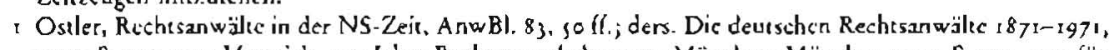
1971, S. 220-103; Hesnrich, roo Jahre Rechtsanivaliskanimer München, München 1979, S. 103-171; für die Strafverteidigung: Ostendorf. Dic Strafverneidigung im Dritten Reich, Der Strafvertcidiger 1983, S. 120fl: Gistrow, Tödlicher Alleag - Serafvertcidiger im Driten Reich, 1981. 
Auch gegenüber der Reichsrechrsanwaltsordnung von 1936 besteht ein ambivalentes

Verhältnis, wenn man darin angebliche anwaltspolitische Fortschritte sieht oder zumindest darauf verweist, $\mathrm{d}_{2} ß$ dic Vorarbeiten hierfür bis in die Weimarer Zeit zurückgingen.

Bildete die Anwaltschaft ein Element des Widerstandes, übernahm sie die Rolle der Mitläufer oder gehörte sie gar, wie die Namen Freisler, Frank und Kaltenbrunner nahelegen, zu den Führern des Terrorregimes? Hat sie ihre Aufgabe technisch forrentwickelc oder ist ihre Rolle politisch zerstört worden?

Die bisher hierzu in der Lireratur gegebenen Antworten befriedigen nicht. Bei Güstrow ${ }^{2}$ heißt es:

"Der Kreis wirklich überzeugter Nacionalsozialisten war verhältrismäßig klein, auch wenn sich nach 933 viele Anwälte aus Opportunismus, Bequemlichkeir, Existenzsorge und Karrierclusi das Parteibuch besorgt hatten oder in Parteigliederungen eingetreten waren. « Er unterscheider die "Heißen «, "Lauwarmen « und die "Kalten * Anwälse im NS-Rechtssystem. Seine Erinnerungen setzen auf dic Einzelpersönlichkeir, ihren Charakzer, ihre Moral, wenngleich er mit dem Begriff der "Existenzsorge* auch anwaltsspezifische Strukturen anspricht. Für Ostler ${ }^{3}$ serlag der deutsche Anwalt in geringerem Maße als seine juriscischen Kollegen der anderen Beruissparten der Infizierung durch den nationalsozialistischen Ungeist. " Feighcit * und "Mut", "aufrechre unabhängige und uncrschütterliche Haltung sind aber auch bei ihm die entscheidenden individuellen Kriterien für das Verhältnis zum NSRegime, wobej er auch auf die „Glückslage des Standes « hin rveist, als sfreier Beruf « nicht so sehr staatlicher Einflußnahme zu unterliegen wie die Richter.

Heinrich" geht in der von Bader übernommenen Gesamteinschätzung darüber hinaus: "Er (der Rechcsanwalt, U. R.) galt als liberalistisch, wenn er die Interessen der Einzelpersönlichkeit vertrat, als gcmeinschaftsfeindlich, wenn cr nicht cat, was die Partei und ihre Amtswalter wollten. Innerhalb der Juristenschaft war er ein Elcment des Widerstands gegen Liebedienerei und Selbstaufgabe. ${ }^{\text {s }}$

\section{Freier Advokat oder Rechtsdiener - Zur Konzeption einer Anwaltsgeschichisschreibung}

In der bisher dominierenden Geschichesschreibung zum Anwaltsberuf vermische sich eine strukcurell orientierte Analyse mit personalistischen Ansätzen, bei denen nicht der Bcruf des Anwalts an sich, sondern die Pcrsönlichkeit, sein Verancworcungsbewußtsein, scin Anstand und gar seine "vaterländische Gesinnung " ${ }^{6}$ Garant für seine Widerstandshaltung gewesen sein soll.

Die historische Betrachtung kann sich dann auf das Erzählen individueller Geschichren beschränken, aus denen dic Anwaltschaft wenig für ihren Beruf, aber viel für ihre innere Einstellung lernen kann. Es können dann sogar gegensätzliche Lehren gezogen werden, wenn die einen dic absolure Freiheit des Berufs als demokratisches Fundament fordern, während andere sittliche und moralische Auslese fordern, um Anwalts»persönlichkeiten« zu gewährleisten.

Gerade der Nationalsozialismus könnte in seiner kompromißlosen Vernichtung aller

2 Gustrow, Tödlicher Allıg, Stralverncidiger im Driten Rejch, 1981, S. izf.

3 Rechusanwäle in der NS-Zeir, AnwBI. 83, 59.

4 Heinrich, a. a. O

, A. a. O., S. 368 .

6 V gl. Osilcr, a. a. O 

scandsporential hat, weit der Gegner der Demokratie sie häufig besser kennr als ilır Anhänger.

Die differenzierte Anwaltspolitik des NS-Staates ist daher nicht Ausdruck von Unentschlossenheit und Planlosigkeit, sondern Indikator dafür, daß im »deurschen Rechtsanwaleu auch andere Elemente enthalien waren als dic von einer "freien Advokatur« erforderten. Diese Elemente mögen es gewesen sein, an die autoritäre Regelungen anknüpfen konnten.

Dies crschließs sich jedoch nur demjenigen, der das Bild der w freien Advokatur a ${ }^{7}$ als Grundlage des demokratischen Rechesstaztes erfaßt und aus dem bis heute schillernden Leitbild des * Rechtsanwalts * herausgearbeitet hat. Die Geschichtsschreibung ist damir uncrennbar mit der Analyse des Anwaltsbildes heute verknüpft.

Dic besonderc Schwäche der Vergangenheitsbewältigung in Deutschland ist bereits begrifflich sichtbar, weil dic "freie Advokatur « sich bis heute hinter einer speziell deuzschen Ydeologie der Verknüpfung von Privatinteresse und Gemeinwohl verbirgt.

Während in den Nachbarländern nach wie vor Anwälte als *Advokaten ", d. h. als die zur Vercidigung der Rechte sHerbeigerufenen a (lac.: advocatus, sollicitare) bezeiclinet werden, ${ }^{70}$ verloren die deutschen Advokacen diesen Tirel 178 , durch die Preußenkönige. Sic wurden zu beamteten Assistenz- oder Justizräten degradiert, später wegen ih.rer Zulassung zum Gerichr (= Recht) als Rechrsanwälte bezeichnet. ${ }^{8}$ Der Advokacenbegriff wurde zum Schimplnamen (vgl. . Winkeladvokata), und aus dem Rechtsanwalt wurde der Anwalt des Rechts, der dem Rechte dient und nicht den Interessen des Mandanten. Die Nationalsozialisten emannten ihn zum Mitglied des Standes der wRechrswahrer, in dem er ein Organ der Rechespllege sei, wie es bereits das Reichsgericht 1925 festgestellt hatte, als es einen KPD-Anwalt vom Prozeß ausschloß.9

Die Bundesrechtsanwalisordnung hat diescr Bezeichnung in $\$$ ein Denkmal gesetzt. Der deutsche Advokat ist ein Organ der Rechtspflege, um deren geordneten Gang zu gewährleisten. Gleichzeicig erfüllt er aber seine Aufgabe, wie $\$ 2$ BRAO feststellh freiberuflich. Er ist ein freier Advokat in samtsähnlicher Stellunga, so das Bundesverfassungsgericht. ${ }^{10} \mathrm{~A}$ s Pflichtverteidiger vor Gericht soll er sich nicht auf seine Berufsausübungsfreiheit berufen können."

Der deutsche Rechrsanwalt bleibr im wesentlichen zugelassen: Zugelassen zum Beruf durch Justizbehörde (und Anwaltskammer) oder, nach ehrengerichtlichen Verfahren, durch den Bundesgerichtshof, zugelassen zu einem Gericht durch den Gerichrspräsidenten, zugelassen zu einem Verfahren durcl den Richter. Während in anderen Staaten die freie Advokatur erkämpfr und durchgesetzr wurde, wurde sie in Deutschland mehr oder weniger s zugelassen . Mal durften bürgerliche Demokraten niche in den Beruf (in Preußen z. B. Theodor Storm), meistens war den Juden der Beruf versperr (bis 1860 und von 1933 bis 1945) und bäufig waren Kommunisten ausgeschlossen (in Preußen vor 1878 , solange sie den Status öffentlicher Bediensteter hatten; behindert in der Weimarer Zeit und in den soer Jahren; geserzlich ausgeschlossen von 1933 bis :945).

7 Vgl. dazu vor allem Gneist, Freie Advokanur. Die erste forderung aller Jusuzzreform in Preußen. 1867.

7a Franz.: avocal: ital.: advocarus; holl.: advocast; engl.: sollicicor.

8 Vgl. Weißler, Geschichre der Rechisanwaleschatt, 1967, Neudruck der Ausgabe von 2905, S. 421 (I

g RG $J W 26,1757$.

$10 \mathrm{NJW} 83,1536$.

ir So BVerG NJW 75 , 10,5 
Während in England die Advokaten den Staat zwangen, zunächst bei seiner eigenen Interessenvertretung im Strafrecht auf die Advokaten als nStaats-«anwälte zurückzugreifen und dann auch die Richter aus dem Stande der freien Advokaten auszuwählen, blieb in Deutschland in Ausbildung und Prozeß der Ricluter das Vorbild und der Aufseher über den Anwalt.

Der Begriff der "freien Advokatur * hat im Geserz keinen Plarz gcfunden. Er wird unzureichend vertreten durch den wreien Berufa in $\$ 2$ BRAO, der nur seinen sozioökonomischen, nicht jedoch seinen politischen Inhalt repräsenciert.

Damit fehlt aber bereits das zentrale analytische instrumentarium, um die differenzierte Herangehensweise der Nationalsozialisten an die deurschen Rechtsanwälce als effiziente und erfolgreiche Strategie zu vcrstehen, bei der man am Rechrsanwalt, am Organ der Rechtspflege und an der freiberuflichen Tätigkeit festhielt, Disziplin und Idcalismus einforderce, gleichzeitig aber alle politischen liberalen Elemente in der Anwaltschaft sowohl personell als auch strukturell vernichtete.

Der deutsche Faschismus zielte auf den politischen Liberalismus, dem man die Schuld daran gab, daB die Arbeiterbervegung eine solche Bedeutung erlangen konnte, daß die Grundlagen kapitaliscischer Herrschaft sowohl ökonomisch als auch polirisch bedrohe werden konnten."

Die freie Advokatur als liberale Speerspicze der bürgerlichen Demokratie im auroritären Rechtssystem mußte damit das wichtigste Angriffsziel in der Anwaltschaft sein.

Wenn die Nazi-Propaganda die von ihr gewollte Ausromung der liberalen Restbestände der freien Advokatur mit einer Politik gegen die "Geldgier der Advokaten«,

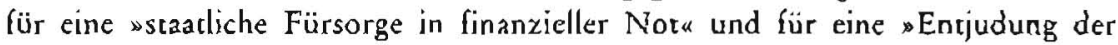
Anwaleschafe $\alpha$ verband, so steckt historisch darin mchr Wahrheit als eine personalistische Geschichrsschreibung hervorbringen kann. Im folgenden soll die Funktion des Geldes und die Rolle der wudenfrage für die Legitimation der Zerstörung der freien Advokatur in Deutschland angedeuter werden.

\section{Freie Advokatur und Geld}

Die private Bezahlung durch den Bürger hatte den Advokaten einst vom Staat befreit und zum Vertreter der Bürgerrechre und der Demokratie gemachr's. Sie hare ihn aber auch in die Arme der Reichen getrieben und dem Volk entfremdec.

\section{a) "Der geldgierige Advokat" - Ideologischer Hebel zur Ungestaltung des} Anwalesleitbildes

Der wgeldgierige Advokat w war ein populäres Zerrbild nicht nur des Kleinbürgerrums, sondern auch in der Arbeirerbewegung, die im Anwaltsstand keine Möglichkeit sah, ibre interessen vertreten zu sehen, ${ }^{14}$ weil der Anwalt für den Arbeiter zu teuer war.

y Vgl. dazu Reifncr, Institurionen des faschisischen Rechtssystems in: ders. (Hg.), Das Recht des Unrechesstastes, 1981, S. 76 ff.; Wahsner, Faschismus und Arbeitsreche, in: Reifner (Hg.) a. a. O. S. go ff.; Rcifncr, Jusriz und Faschismus, in Reifner/Sonnen (Hg.). Seraffusciz und Polizei im Drinon Reich, 1983; Mason, Sozialpolitik im Driten Reich, 1978.

13 Vol. schon dazu Feuchewanger, Die Freien Berule, 1922, S. 16s f.

is Ausführlich hicriber habe ich in Blankenburg/Reifner, Rechesberaung-Rechesprobleme durch soziale Definition, 1981 , S. 163 ff. bericheer. 
Beide Kritikansäıze uncerscheiden sich jedoch ganz grundlegend. Die Arbeicerbewegung begrüßce gerade die politische Dimension der freien Advokatur, da ihre Interessen zu Rechten transformiert in einem fairen Verfahren ins Rechrssystem integriert werden mußcen und gleiches Recht auch als Schutzschild gegenüber sraatlicher Willkür zur Unterdrückung kollektiver Aktionen notwondig war.

Allein schon die Tatsache, daß die Arbeicerbewegung sich in Ferdinand Lasa!le, Karl Liebknecht und Paul Levi Anwältc zu ihren Führern auserkor, macht deurlich, daß das Wort vom "geldgicrigen Advokaten * eine ökonomisch verkürzte Sicht der Rolle der Anwaltschaft implizierte." Entsprechend organisicrec die KPD auch in ihrer Massenorganisation "Rote Hilfe" ein ökonomisches Versicherungssystem. das die Beschäftigung von I $_{3}$ Rechtsanwälcen für die Arbeiterbewegung garantierte, Die Gewerkschafien hatcen neben ilirem System der Arbeicersekretäre im SyndicusAnwalt ebenfalls ein kollektives staats- und gegnerfreies Finanzierungssystem geschaffen.

Gerade diese Systeme wurden von den Narionalsozialisten (unter dem Beifall einer kurzsichtigen Anwaltschaft) sofort zerstört.

Mit der Formel von der "Geldgier" sollte auf der Rechten die Bezichung des Anwalts zu seinem Mandanten gelöst werden, damit er vom Anwalt der Rechre des Bürgers zum Anwalt des Rechts des Staates werde. Deshalb erscheint in all dieser Kritik, ob sie nun von der Richterschaft Anfang des Jahrhunderts geäußert vurde, ${ }^{16}$ oder ob sie die Nazi-Kampfschrifc „Der Stürmer" artikulierte oder ob die "gelehree» Jurisprodenz sie in wohlgeseczten Gedanken konstruierte, als Alcernative des geldgierigen Advokaten immer das staatstreue Organ der Rechrspflege. " Er ist der Kooperacionspartner des Richters, der völkisch gesinnte Kamerad oder der wahrheitsliebende Betreuer und Ermahner des Mandanten, und zwar dort am scärksten artikuliert, wo der Staar sich prinzipiell in Frage gestclle siehe und, wie im Straf- und Arbeitsrecht, gegen Staats- und Syscemfeinde die Gerichre mobilisiert.

Der ideologische Daucrangriff gegen die freie Advokatur wurde nicht nur mit Hilfe des Geldthemas, sondern auch auf andere Wcise geführr. Mir der beinah traditionelIen Identifikation der Geldgier mit der jüdischen Menralität ${ }^{1 \S}$ konnte man alle Liberalitär und damit auch die freie Advokacur rassistisch bekämpłen, um über den "jüdischen Welrbolschervismus a den idcologischen Zirkel zu schließen. Antisemitismus war damic vor allem auch eine Politik des rcakrionären Anti-Liberalismus. Die Daverhetze in "Stürmer" gegen die wjüdischen Advokaten * war ein solcher rassistisch verschärfter Anti-Liberalismus: "Wenn er hundertmal weiß, daß der Prozeß verloren ist, macht er den Klienten mir seinem jüdischen Wortschwall so besoffen, daß dieser von dem Erfolg überzeugt ist und einen beträchtlichen Vorschuß leistec. "Auf der anderen Seite werden freisprechende Urteile der Gerichte mit ihrer „Verjudung * begründec. "Eine solche jüdische Sauwirtschaft sollte man gar nichr für möglich halren. a'g

Das "Jüdische besteht zum Leidwesen des nStürmers « nicht in der Erfolgslosigkcit jüdischer Rechtsanwälce, sondern darin, daß er die "vom gesunden Volksempfinden

is Locwenteld, Bemerkungen zur Diskusson uber die Rechesvertretung und Anwaltschuft, Dic Justiz Bd. VII, S. 446 ff., gegen das Mißversiandnis bei Heindi, Rechesvererecung und Anwalischaft, Dic Justiz Bd. VIII, S. 339 If,; zur Diskussion auch Ostler, Die deueschen Rechesanwaltc, a. a. O., S. 220 I.; Levin. Rechesverteteung und Anwaltschalt (cinc Erwiderneng), Dic Justiz. Bd. VII, S. $35 s$ ff.

$16 \mathrm{Vgl}$. dazu Ostler, Die deueschen Rechtsanwälec, a. a. O. S. 129 ff.; Feuclsturanger, Die freien Berufe, 1922, S. sis l.

17 Auf einen schr prinzipiellen und einfachen Nenner gebrachs in dern A rikel r Recheswahrer oder bezahlter Kulik, Das Schwarze Korps (Zeitung der SS) v. 5. 8. 1937, S. 6/Folge 31.

18 So schon Weißler, Geschichte der Rechtsanwaltschalt, (190\%) 1967, S. 603.

ig Fall des Ofenjuden Gassenleimer “, in Der Stürmer Nr. 19 vom Mai 1931, S. 2. 
verlangten Urteile « mit dem Recht zu verhindern sucht. "In unseren Gerichıshäuscrn (ist) nicht mehr die vergeltende Gerechrigkeit, sondern der leblose blöde Paragraph besrimmend, der dem Juden als Werkzeug dient. ${ }^{20}$ Man wünsche sich neine Revolution, dic auch einmal mit dem jüdisch-römischen Paragraphenwust aufräumt und die an seine Stelle setzt das heilige deursche Recht. ${ }^{21}$

Die Inanspruchnahme von Verfahrensrechren im Strafprozeß, die sich vor allem auf die liberale Unschuldsvermutung gründen, wird vom «Stürmer * so beschrieben: "Mit jedem Raffinement werden ihm (dem Richter, U. R.) Steine in den Wcg gelegt, über die er stolpern soll. Nichts wird unversuche gelassen, ihn unter geschickter Spekulation auf menschliche Schwächen zu irgendeiner kleinen Dummheit zu verleiten. Wenn dem Juden dies gelungen ist, dann wird der Richrer ... dem jüdischen Willen gefügig gemacht. "s So findet es der "Stürmer a auch unglaublich, daß ein (narischer ") Anwalt vor Gerichs von der „Unschuld « und von seinem Mandanten spricht, statt von "Nationalsozialismus und Führer! «³

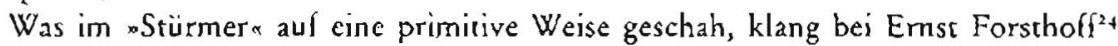
gebilderer: „Eine Bewegung wie dic liberale, die sich das ammaßende und täuschende Ziel einer Vergeistigung der Politik setzte, die das politische Leben in cine endlose Diskussion aufzulösen strebte (...), die die Macht als roh, ungcistig, menschenunwürdig diskredinierte, konnce niemais wirkliche Volksbewcgung werden. ... Das Aufkommen der proletarischen Bewegung... ist das deutliche Kennzeichen des Volksverfalls... . Das bürgerliche Zeiralser vird liquidicrt, und es ist die Verheißung einer besseren Zukunft, daß es mit rücksichesloser Entschlossenheit und dem Mur zur äußersten Konsequenz geschieht. “

Rechtsanwalt Pror. E. Noack, Präsident der Reichsrechtsanwaltskammer und auch hcute nocly zitierter Kommentator der RRAO von 1936, beginnt scinen Aufsatz über "Der freic Anwalt im Dritien Reichass mit dem Geld-Thema und wendet sich aber dann dazu, den Anwaltsberuf in einer nidealistischen Welranschauung $\alpha$ so zu definieren, daß das Band zu den Rechten des Mandanten aufgelöst ist. Am Beispiel des gegenüber dem Anwalt geständigen Verbrechers führt Noack aus: "Die ganze Frage (ob Niederlegungspfliche besteht, U. R.) ist erlcdigt, wenn man dem Standpunkt folgt, daß es überhaupr nur Interessen der Gesamtheir im nationalsozialistischen Stazte gibr... Es wird mir dann jeder zugeben, daß der Anwalr in diesen Falle, gerade weil er fremde, im Widerspruch zu den Belangen der Gesamtheit stehendc Interessen niche vertreten darf, niederlegen muß.... Freilicit im Wollen und Handeln isc begrenzt durch die übergeordneten Lebensrechre des Volkes. . . Die Existenz (des Anwalrs, U. R.) isr Dienst an der Deutschen Volksgemeinschafr, also alles ist nur Mitcel zum Zweck, und der letzte Zweck ist immer Dienst am Volke ... und daher kann Recht nur das sein, was dem Volke dienr, und damic kann jeder Rechtswahrer nur am Rechtsdienst als Mittel zum Zwcck gebunden sein. ${ }^{26}$

\footnotetext{
20 Der Stirmer a. a. O.

2 I Der Sturmer Nr. 21 von Ma1 1913, S. 1.

22 Der Stürmer Nr. 3 vom Jan. 1933. S. 6.

23 Der Stürmer Nr. 19 rom Mai 1936 S. 1-7 über dic Verhandlung gegen Heymann wegen Weinbetrug.

24 Der coule Strat, 1933, S. 16 I.

$25 \mathrm{JW} 36,1746$ If.

26 Wie wenig manches uberwunden isı, zeigr Schwinge, Richer und Strafvereidiger in den USA, DR Z 76 , 300 (304); vgl. auch Schwinge/Zimmerl, Wesinsschau und konkrctes Ordnungsdenken im Strafrecht, 1937.
} 
Die Befreiung der Advokatur vom Staat durch die private Bezahlung zeigte sich mir Beginn der industricllen Revolution auch als Fessel in Gestalt materieller Sorger. Die Zahi der Anwälce wuchs über die finanzkräfrige Nachfrage hinaus.

In der nachfolgenden Tabelle zeigt sich eine Zunahme der Anwaltschaft von rgos bis 1933 um 146\%. Gleichzeitig fiel das Verlıälınis von Bevöllkerung zu Rechtsanwälten un dic Hälf́re.

Anwaleszablen oon 1905 bis 1933

\begin{tabular}{llllll}
\hline Jalur & $\begin{array}{l}\text { Anzahl der } \\
\text { Anwälte }\end{array}$ & $\begin{array}{l}\text { Einwohncr } \\
\text { pro Anwalt }\end{array}$ & Jahr & $\begin{array}{l}\text { Anzahl der } \\
\text { Anwälte }\end{array}$ & $\begin{array}{l}\text { Einwohner } \\
\text { pro Anwali }\end{array}$ \\
\hline 1905 & 7835 & 7194 & 1921 & 12276 & 5397 \\
1907 & 8608 & 6548 & 1923 & 12729 & 4702 \\
1909 & 9578 & 6331 & 1925 & 13578 & 4606 \\
1911 & 10817 & 5606 & 1927 & 14963 & 4171 \\
1913 & 12237 & 5280 & 1929 & 15881 & 3990 \\
1915 & 13024 & 4985 & 1931 & 17220 & 3624 \\
1917 & 12393 & 5239 & 1933 & 19276 & 3374 \\
1919 & 12030 & 5397 & & & \\
\hline
\end{tabular}

Wie wenig diese Zahlen etwas úber die Grenzen des Anwaltsberufs aussagren, hat Magnus ${ }^{27}$ durch den Vergleich mit anderen Staaten wie USA, Frankreich und Italien gezeigr, wo eine drei- bis zehnfache Anzahl von Anwälcen pro Einwohner existierte. Gleichwohl war die nach r 870 aus staatlicher Bevormundung und Fürsorge entlassene freie Advokatur auf diese Herausforderung nicht vorbereitet. Ihr Berufsbild war von der richtergleichen Ausbildung her über das prozeßorientierre Gebührensystem alizu stark verengt auf die forensische Tätigkeit. Eine Ausweitung der Beratungs- und außergerichtlichen Gestaltungsfunktionen mit entsprechender Neugestalrung von Aus- und Forrbildung in die ökonomische und soziologische Disziplin hinein, ${ }^{28}$ eine neue anwaltliche Preispolitik sowie eine Trennung von kollekriver ökonomischer Einkommenssicherung von der persönlich freien anwaltlichen Tärigkeir als Alternative zum staatlich verordneten Gebührensystem, ${ }^{29}$ die Einfübrung der Fachan waltschafr ${ }^{\circ}$ oder weitergehende kollekrive Vercretungsmodelle" wurden nur von einer Minderheir ernsthaft ins Auge gefaßt.

Die Mehrheit der Anwälre suchce eher nach Lösungen innerhalb des fremdbestimmten Rahmens und verfiel dabei zunehmend auf den Scaacsschurz für die Anwaltschaft. IgI I wird der numerus clausus noch einmal abgewehrt.'2 Ab ig2s zeichnet sich jedoch deurlich eine Richrungsänderung ab.

Unzählige der freien Advokatur hohnsprechende Vorschläge wurden gemach, mit dem einzigen Ziel, die Zahl der Anwälte zu beschränken. Die Fróntbewährung im ersten Weickrieg solice dabei ebenso wie Kinderlosigkeit, Geschlecht und Alter eine Rolle spielen. ${ }^{33}$ Die Forderung nach dem numerus clausus wurde auf der Abgeord-

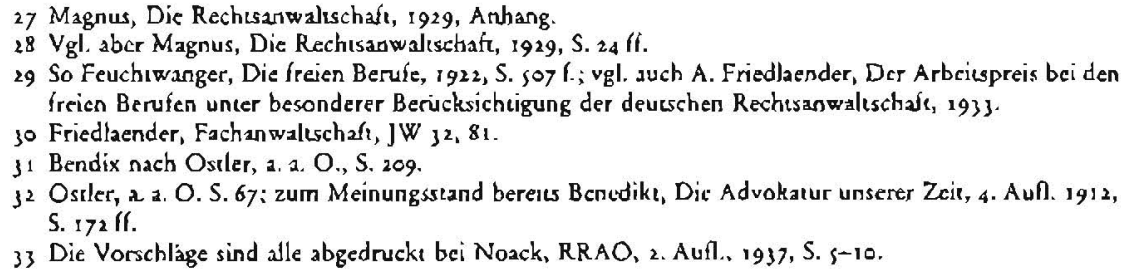


netenversammlung vom März 1930 zunachst für Referendare beschlossen. 1932 hieß cs schließlich im Anwaltsblatt: "Wenn nicht alles täuschı, nähern wir uns mic riesigen Schritten einer Zeit, in der keine Halbheiten mehr gelten. Denn die allgegenwärtige Nor ist nicht mit Halbheiten zu meistern, sondern eben nur mit extremen

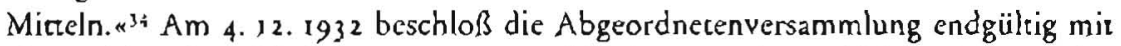
überwältigender Mehrheit zum ersten Mal in der Anwaltsgeschichte, einen totalen numerus clausus zu fordern. Der 1932 gewählte DAV-Präsident Dix verlangte dann am 8. 2. 1933 von der neuen NS-Regierung bei ihrem Justizminister Gürnner hierzu eine Notverordnung.

DAV-Präsidiumsmitglied Jessen, der die Verhandlungen des DAV mit dem Verein der gemeinnützigen Rechtsauskunírsscellen über die Beteiligung der Anwälte an der öffentlichen Rechtsberatung $193^{\circ}$ zum AbschluB brachce, propagierce als neves Anwaltsbild den $»$ Rechtsfriedensanwalta, der streitvermeidend die Armen beraten sollte." Nach 1933 entwickclce er diese Ansätze weiter zur NS-Rechtsberreuung"sa, in der der Anwalt zum staatlichen Befriedungs- und Repressionsinscrumenr herabsank. Weitere Mittel zur Einkommensverbesserung sollte dann die Ausdehnung des Anwaltszwangs auf alle Gerichrszrveigc sowie dic Einführung eines allgemeinen Rechtsberatungsmonopols sein. Alle diese Aufgaben mußte der Scaat erfüllen, zu dessen Kontrolle die freie Advokatur einsumals vom Bürgertum entwickelt worden war.

Der NS-Staat nahm dieses Angebor gerne aul und fügte weitere Maßnahmen zur Verstaatlichung des Anwalusberufes hinzu.

Am 7. 4.1933 erging das Gesecz, wonach Anwälte, dic sich sim kommunistischen Sinne betätigt « hatten sowie Anwälte jüdischer Abstanımung von der Zulassung zu diesem Beruf ausgeschlossen waren. ${ }^{3 s b}$

Gleichzeitig wurden mic Ausnahme von "Fronckämpfern « für is 00 Anwälce aus polirischen und rassischen Gründen die Zulassungen zurückgenommen. Am 20. 7. 1933 wurde eine Alrersgrenze für Anwälte eingeführt und "Maßnahmen gegen das Doppelverdienercum e eingeführt. ${ }^{36}$ Ein Verbor der Syndici-Anrvälte erfolgce jedoch nicht. Ab Juli 1933 wurden praktisch auch keine Frauen mehr zum Anwaltsberuf zugelassen. Im Dezember wurde dann die Zulassung zur Anwaleschaft entsprechend den beameenrechrlichen Treuepflichten ausgestatter, wonach ^die Persönlichkeit des Antragstellers nach seinem bisherigen Verhalten ... Gewähr für zuverlässige Berufsausübung ... * bieten soilte, "7 was nach Auffassung der Rcichsfachgruppe Rechesanwälte im Bund Nationalsozialistischer Deutscher Juristen eine Maßnahme gegen volks- und staatsfeindliches Vechalten war. Gleichfalls im Dezember setzte man den Anwaleszwang auf Streitwerte über s00 RM aucb vor den Amtsgerichten fest, kürzte aber gleichzeitig die Armenrechtsgebühren um $5 \%$.

Obwohl 19341364 Anwälte neu zugelassen wurden, ${ }^{38}$ was bei 5900 Assessoren allein in Preußen nicht besonders viel war, sank die Zahl der Anwälce bis 1935 auf 18712 . 1936 tracen daun das Rechtsberatungsmißbrauchsgeseiz, das die mit Berufsverbo: belegten Anwälte endgültig von jeder rechtlichen Tätigkeit ausschloß und den verbliebenen "deutschen "Anwälten das Rechrsberatungsmonopol (bis heure) sicherte, ebenso in Kraft wie die neue Reichsrechtsanwaltsordnung, die mir Ausleseprinzip,

14 Zitierr nach Osiler, a. a. O., S. 1156.

3) Jessen, Rechusiürsorge und Anwaleschafh, Die Reclusauskunfe 1932, S. 32 ff.

$35^{2}$ Jessen, Die Rechesbetreuung der deutschen Anwalschaft, Die Rechesauskunt 1933, S. 125.

3) b RGBl. I, I 68 .

36 RGBI. I s 12.

37 RGBI. 11258

38 Akten des Reichsjuscizminisceriums im Bundessrchiv Koblenz R 22/251, S. 76. 
Partciaufsicht und Führerprinzip die Verstaaclichung des Anwaltsberufs weitgehend Pestschricb.

1938 waren schlicßlich alle Anwälte jüdischer Abstammung mir Berufsverbor belegt, nachdem sie vorher von der übrigen Anwaltschaft allmählich durch das Vercrecungsverbor für »Arier «, durch die Zwangsauflösung von Bürogemeinschafren mit sarischen a Anwälten ab 1933 isoliert worden waren. Da die Reichsrechtsanvaltskammer am 2. 7. 1934 noch einmal das ehrengerichtlich festgelegte Verbot der Enrgeltnahme für den Praxisverkaul aus standesrechthichen Gründen bekräftigte, ${ }^{39}$ war außerdom gewährlcistet, daß dic verblicbene Anwaltschaft sich an diesem bruıalen Vorgehen entsprechend bereichern konnte.

Alle diese Maßnahmen haben lerztlich wirtschafulich den "deutschen a Anwälten nichts genützt. Zwar wurde hierdurch die Zahl der Anwälte von 19276 im Jahre 1933 auf 14 Soo im Jahre 1939 reduzicrt. ${ }^{40}$ Das Durchschnitseinkommen sticg jedoch trotz wirtschaftlichem Aufscliwung von $9490 \mathrm{RM}$ im Jahre 1933 nur auf ro 849 RM im Jahre 1936. Der Stand von 1929, wo ohne Berufsverbore und numerus clausus das Durchschniteseinkommen noch 18310 RM betrug, ist nicht im entfernresten mehr erreicht worden.

Ministerialrac Jonas vom Reichsjustizministerium schrieb Anfang Juni 1935, daß "die Verdienstmöglichkeiten der Anwaltschaft ... noch niemals so ungünstig gewesen (seien) wie jetzru. Dies sei weder nkanjunkturbedingt " noch "vorübergehend $\alpha$. Eine "Ankurbelung des juristischen Konsums a käme nicht infrage. "Muß man sich danach auf das Zuzugsproblem beschränken “."

Die Anwälte haben sich in einzelnen Fällen gewehrt, wie z. B. die Brief- und ArtikelFlut, die 1935 zur Wiederaufhebung der Altersgrenzc für den Zwangsruhestand führte sowie die Brielkampagne der Syndici-Anwälte, die effekriv ihren Ausschluß aus der Anwaltschaft verhinderte, zeigt...12 In den entscheidenden Punkten waren sic jedoch kurzsichrige Kleingewerberreibende, die die freic Advokarur für ein vermeintliches Linsengericht opferten. Davon zeugen z. T. erschrcekende Briefe von Anwälten, in denen dem Reichsjustizministerium vorgerechnet wird, wieviele jüdische Anwälte noch in der Stadt praktizierten und den "Deutschen* die Mandace wegnehmen. ${ }^{43}$ Der anwaltliche Bourgeois haste den anwaltlichen Citoyen wverkaufto und sich damit lecztlich auf lange Sicht doch seiner eigenen wirtschaftlichen Grundlage beraubr.

Weic verheerender aber für die freie Advokatur war es, daß der Anwalt nunmehr freiberuflich Verdiencnder von staatlichen Gnaden war. Dicjenigen, die dies akzeptiert hatcen, konnten kaum Argumente gegen die nunmehr geforderte "Treue zu Fuhrer, Volk und Staat $\alpha$ zum "Dienst am Recht a." vorbringen.

39 H V Ziff. 62 der Richelinien für den Anwalesberul, Bundesarchiv $\mathrm{R}_{43} 1 \mathrm{l} / 1534, \mathrm{~S} .74-8 \mathrm{r}$.

$40 \mathrm{Vgl}$. Reifncr, Institucionen des faschistischen Rechessystems, in ders. (Hg.), Das Recht des UnreclusstaaLES, 1981, S. 26

11 Jonas, Zur Nollage der Antualuschaft - Secllungnahme xu Noack -, Internes Papier des RJM R 22/251 Bl. 105 a Bundesarchiv.

42. Briele in den Akecn des RJM in Koblenz.

43 Bricle in den Akten des RJM im Bundesarchiv Koblenz von RA Hercher vom 30.4.34 (R 22/263), RA Noack an Freisler vom 30.12. 34 (R 22/26), RA Dr. Birkholz (R 22/251 Bl. 60).

44 So der Titelaufsacz des Reichsrechisiühecrs RA Racke zur neuen RRAO sowie zum RBerMiBG in JW 1936,16 . 
Im Jahre 1933 waren nach den offiziellen Nazi-Seacisciken von $19500 \mathrm{im}$ Deurschen Reich zugelassenen Anwälten 4394 Anwälce jüdischer Abstammung. "s 1935 waren es noch $2550^{46} 1937$ nur noch $17533^{47}$ und nach dem 13.8. 1938 gab es keinen prakrizierenden jüdischen Rechtsanwalt mehr in Deutschland. Im Bcreich der Berliner Anwaltskammer waren $193360 \%$ der Anwälte jüdischer Abstammung, in Wien waren es 1938 sogar $80 \%$. Der Anteil der Deutschen jüdischer Abstammung an der Gesamrbevölkerung in Deurschland lag damals unier $3 \%$, während er bei der Anwaltschaft $22 \%$ betrug.

War dieser Anteil zu hoch und die deutsche Anwaitschaft, jüdisch unterwandert, wie es auch nicht der NSDAP nahestehende Konservative giaubten? Um dieser Idcologie entgegenzutreten, reicht es nicht, die Zahlen der Nationalsozialisten einfach zu referieren oder gar darauf hinzuwcisen, daß jüdische Anwälte auf jeden Fall in der Minderheit blieben..$^{8}$ Auch hier geht es zunächst um dic Berichrigung einer falschen Fragestellung, die, statt nach der Bedeutung der jüdischen Anwälte für die Entwicklung der freien Advokatur und damit für die Demokrarie in Deutschland zu fragen, sich in der Mitteilung der Zahlenverhältnisse sowie der Abscammung einzclner Repräsentanten die NS-Einteilung unbewußt unterschieben läßt.

Dann wird der wdeutsche * Anwalt heute, der sich für den Kampf für mehr Demokratie und damit beruíspolitisch für die freie Advokatur entschlossen hat, feststellen, daß er sich in die Tradition der jüdischen Advokaten in Deutschland zu stellen hat, dic den Platz der Liberalitä und des Fortschritts im Rechessystem (keineswegs nur freiwillig) cingenommen hatten.

In Preußen hatte sich das feudale Religionsmonopol so ausgewirkt, daß man den Staatsdienst vor den bürgerlichen Idealen vollkommen verschließen konnte, so daß den Juden erst 1840 de jure und $a b 1860$ auch de facro der Zugang zu öffentlichen Ämtern offen stand. Da der Anwaltsberuf bis 1878 in Preußen als öffentliches Amt unter staatlicher Aufsicht organisiert war, waren jüdische Intellcktuelle bis dahin fast vollständig von allen juristischen Berufen ausgeschlossen. Als bereirs 1880 der öffentliche Dienst und insbesondere die Richterschaft für jüdische Deutsche wieder gesperrs wurde, blieb die Anwaltschaft für Juden das einzige jurisrische Betätigungsfeld. Auf der anderen Seire hatren die Preußenkönige gerade innerhalb der deutschen Advokaten immer wieder durch Berulsverbote, Verfolgungen und drastische wAuslesex eingervirkt, so da $B$ von der Preußischen Anwaltschaft in der ersten Hälfte des 19. Jahrhunderts keine Impulse für die freie Advokatur ausgingen. ${ }^{99}$

Verdankten somit die jüdischen Dcutsehen ithre Zulassung zur Anwaltschaft einer polirischen Liberalisierung, so mußren sie gerade von den preußisch-deurschen Anwälten um so mehr sich abheben, als diese in dem ständigen Auslese- und Disziplinierungsprozeß beruflich stark der feudalen Prokuracur angenähert waren und die Gedanken der freien Advokatur eher ins Exil der USA als nach Deutschland getragen hatten.

\footnotetext{
$45 \mathrm{JW} 33,2956$.

$76 \mathrm{JW} 36,562$.

$47 \mathrm{JW} 38,574$.

$48 \mathrm{Vgl}$. xuletzı Ostler, Rechisanivälec in der NS.Zeil, AnwBl. 83, 50 ff., 52; Heinrich, 100 Jahre Rechısanuvalıskammer Munchen, 2. a. O., S. III, meine sogar, den Nazis widersprechen zu mussen, das die Rechwsanwaltskammem wunter jüdischem Einfuß “ gesıanden häten, weil in München nur 1/, des Vorstandes judisclic Ainwalic waren.

49 Vgl. zum Gazzen Weißler, Gischichtc der Rechtsanwaleschaft, S. 467: Feucherwanger, 2. 2. O, S. 165 ff.
} 
Es war somic ein historischer Glücksíall für das in der politischen Demokratie außerordentlich rückscändige Deueschland, daß gerade in den für die Enrwicklung des Rechtsstaates so wichtigen Anwaltsberuf verstärkt jüdische Deutsche eintraten und die freie Advokatur unbeschwert von ihrer Preußischen Deformation misenerwickeln halfen. Bis heure har allein Hugo Sinzheimer das Verdienst der jüdischen Juristen für den Liberalismus in Deutschland vom Exil her dokumenciert. ${ }^{30}$

Für dic Anwaltschaft fehle diese Geschichre der jüdischen Juriszen noch, obwohl, wie hier nur angedeuter werden kann, sie zugleich die Geschichte der freien Advokatur in Deutschland wäre. In ihrer kurzen Tätigkeit im Anwaltsberuf von 1860 bis 1933 haben die Anwälte jüdischer Abstammung die Selbstorganisation der Anwälte, das Standesrecht und die Publiziscik wesentlich beherrscht. Adolf und Max Friedlaender baben 1907 den ersten und auch einzigen Kommentar zur Rechisanwaltsordnung geschrieben und in drei weiteren Aufiagen auf einen Scand gebrachit, der es den Nazis bis 1935 rein technisch unmöglich machte, auf dieses «jüdische Werk zu verzichten. Der 150 -Seiten Kommentar von Noack aus dem Jahre 1934 hacte das umfassende Werk nicht ersetzen können. Mir seinem Gutachren zum Anwaltstag Igrl hatte Friedlaender auch den numerus clausus abgewehre. ${ }^{\text {soa }}$

Kommentare zur Anwalisgebührenordnung und zum Gericheskostengesetz vervollständigıen seine Bedeutung für die rechtliche Verfassung einer liberalen Anwaltschait." Von nicht geringerer Bedeucung war Hachenburg für die deutsche Anwaltschaft. Schon in seinem ersten Staatsexamen hatte er mit erstaunlichem Selbstbewußtsein einem Prüfer, der Daten abfragte, entgegengehalten: "Ich bedaure, ich habe mein Studium nicht im Auswendiglernen von Geserzesdaten gesehen. . ${ }^{\text { }}$ Im Vorstand des badischen und dann des Deutschen Anwalisvereins, als HGB-Kommentator und vor allem als Herausgeber der Jurissischen Wochenschrift und Verfasser der "Juristischen Rundschau * in der Deutschen Juristenzeitung" war Hachenburg anwaltspolitisch prägend gewesen. Dies gile nicht minder für Martin Drucker, der von 1924 bis zu seiner Ablösung durch Dix im Jahre 1932 Präsident des Deutschen Anwaltsvereins war und noch r93 I unbeirrt von der gegenteiligen Mehrheitsmeinung im DAV, vor allem gegen Dix, erklärte, daß der numerus clausus »das unfehlbare Mitrel zur gänzlichen Zerserzung des Anwaltsstandes« werden müsse."4 Der Kampf gegen den numerus clausus ist ganz eindeutig eine Aufgabe der Anwälte jüdischer Abstammung gewesen, welche unterschiedliche politische Auffassungen sie auch haben mochren. Hachenburg,"s Drucker, ${ }^{56} \mathrm{M}$. Friedlaender, ${ }^{57} \mathrm{~A}$. Friedlaender, ${ }^{8}$ S. Feuchtwanger" $"$ und Magnus ${ }^{60}$ scanden vor allem den »deurschen "Anwälten gegenüber, die sich im DAV durchzusetzen vermochten und 1932 mit Dix als neuem -Präsidentẹn einer N.C.-Anwaltschafte den Untergang der freien Advokatur zulieBen. Die Liste der jüdischen Berichterstatcer, Gutacbter und Referenten der Deutschen Anwaltstage und der Themen macht ihre Bedeutung gerade für den Beruf und

so Sinzheimer, Jüdische Klassiker der Deutschen Rechtswissenschaft, 1933 (Ersedruck 1937 in Holland). soa M. Friedlaender, Gutachten für den XX. Deutschen Anwalistag, Beilage zur JW $199_{1}$.

s1 Vgl. Osder, Die Deueschen Rechesanwiàle, 1 \$ $71-1971$, S. 250.

S2 Hachenburg, Lebenserinnerangen cines Rechesanwales, 1927, S. ${ }_{4} 8$

s) Vgl. Osder a. a. O., S. 136 .

is Vgl. Osder 2. 2. O., S. 134 if.

is Hachenburg. Juristische Rundschau, in: DJZ 29, \&\$1.

i6 Drucker, Der Weg der Anwaluschaf DJZ, $31,257$.

$57 \mathrm{M}$. Friedlaender, Justizreform und Anowisnot, LZ 28, 1009.

s8 A. Friedlaender, Numerus Clausus, Betrachtungen eines Verwaleungsjuristen, Die Justiz Bd.IV. S. 92 if.

59 S. Feuchrosnger, Die Freien Berufe, 1921, S. 147.

60 Die Rechtsanwaltschaft, 1929, S. 23 ders. Die Noulage der Anwaleschafi, 1930. 
die Fortentwicklung des im feudal-bürgerlichen Deutschland unterentwickelten Verfahrensreches deurlich. boa

Aber nicht nur im Berufsleben, sondern überall dort, wo es um die soziale Funktion der freien Advokatur ging, die Feuchtwanger damit umschricb, "dem Rechr gegen das Unrecht, dem Schwachen gegen den Srarken beispringen ${ }^{6 r}$, finden sich die jüdischen Advokaten.

Der Strafprozeß is geprägt von Strafverteidigern rvie Paul Levi, ${ }^{62}$ Harry Litten und vor allem Max Alsberg, dessen Anerkennung weit über die deutschen Grenzen hinaus reichte und dessen Schriften und Anmerkungen zum Scrafprozeßrecht und zur Strafverteidigung das Rechtsleben dort prägte, wo es nicht von der rechts-konservativen Strafrechisprofessorenschaft beherrscht war. ${ }^{63}$

Im Arbeitsrecht haben Fraenkel, Sinzheimer und Neumann in Zusammenarbeit mit den Gewerkschaften das liberale und soziale Gegengewicht zum »Arbeitsrechtskarrell ${ }_{*}$ um Nipperdey, Hueck, Dierz u. a. gebildet. ${ }^{\sigma_{4}}$

Es waren aber nicht nur die Kernbereiche des liberalen und sozialen Rechtsstaates, denen sich fast ausschließlich jüdische Anwälte widmeten. Auch in der Mechode gingen sie neue Wege, die bis heure nicht wieder erreicht wurden.

So schrieb Sigbert Feuchtwanger eine umfassende Soziologie der freien Advokacur, ${ }^{\text {ss }}$ in der vom theoretischen Konzept der freien Advokatur her alle auch heuce noch akcuellen Fragen der Anwaltschaft abgehandelt werden. Julius Magnus faßte zum w fünfzig)ährigen Bestehen der freien Advokatur im Deutschen Reich a im Jahre 1929 48 Länderberichte über das Berufsbild des Anwalts in der Welt zusammen und wählte damit die intemarionale Rechtsvergleichung als Mitrel zur Stüzzung der freien Advokatur.

Fraenkels "Soziologie der Klassenjustiz ${ }^{66}$ und Sinzheimers rechtssoziologisches Forschungswerk sind Klassiker rechtssoziologischer Arbeit in Deutschiand geworden. Hinzu kommt das praktisch politische Engagement der jüdischen Advokaten erwa im Republikanischen Richterbund, aus dem beispielhaft nur Namen wie Hirschberg, Rosenberg, Fraenkel, Sinzheimer und Loewenfeld enwähnt werden sollen. Vor allem aber in den Anwalesvercinen und in den Kammem taren sich die jüdischen Anwälte besonders hervor. In München war mit den Anwälten B. Mayer, Schülein, S. Feuchtwanger, C. Oesterreich und R. Held ein Drittel des Kammervorstandes jüdischer Abstammung. In Berlin wurde die Metrheic des Kammervorstandes von jüdischen Anwälten gebildet. Von 25 Vorstandsmitgliedern im Deutschen Anwaltsverein waren r $x$ jüdischer Abstammung, darunter auch ihr Vizepräsiden Heilberg, nachdem Drucker den DAV von 1924-1932 geleitet hatre. ${ }^{67}$

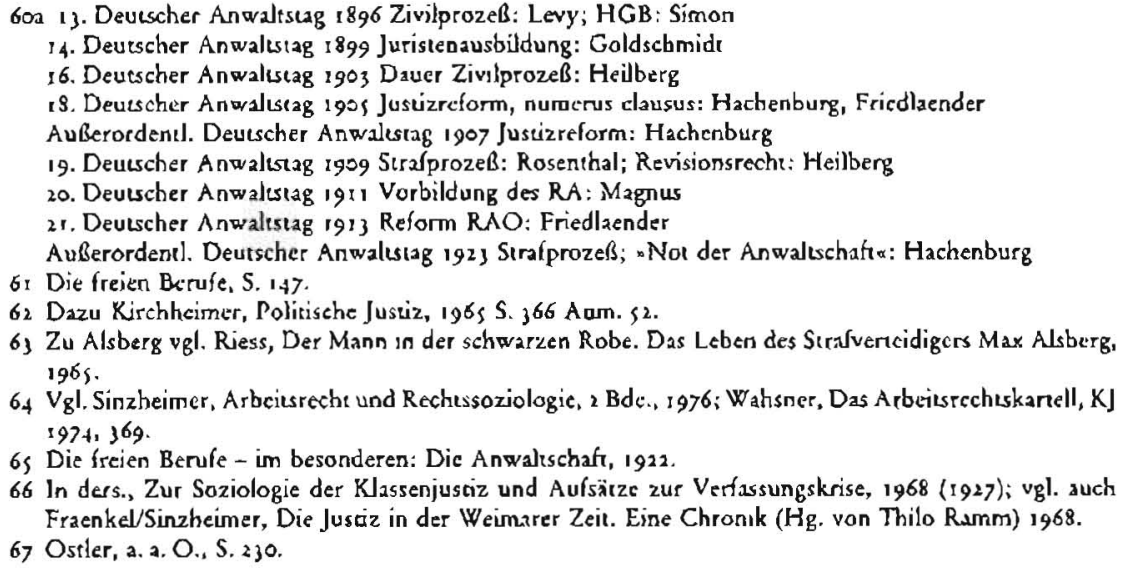


Dic große Lücke, die der Faschismus in die Anwaltschaft gerissen hat, zeigt sich auch in ihrer Geschichtsschreibung über die Entstehung der freien Advokacur im 19. Jahrhundert. Grundlegend isc immer noch die •Geschichte der Rechrsanwaltschalta des ständisch bormierten Anron Weißler aus dem Jahre 1905, der in der öffentlichen Prokuratur das Vorbild des Deutschen Anwalts erbolfite.

Während die jüdischen Juristen, wie der Untertitel bei Magnus ${ }^{6 s}$ bereits deutlich machr, sich bewußz waren, daß die zarte Pflanze der freien Advokarur in Deurschland kaum eigene Gesclichte aufzuweisen hatte und im wesentlichen emigrieren mußte, geht für Weißler die Geschichte der Rechtsanwaltschaft bis auf einen Befehl König Karls in einer Sage zurück. Alles, was in der Feudalzeit an Gerichtsdienern und Vorsprechern, an Prokuratoren und Fürsprechern erscheint, wird in die Geschichte der Anwaitschaft kririklos eingeordnet. In diesem vielzitierten Buch, das im Gegensarz zu den cinschlägigen Arbeiten jüdischer Anwälte 1967 unverànder wiederaufgelegt wurde, finden wir auch bereits die Bemerkung über den schädlichen Einfluß der jüdischen Juristen in der Anwaltschafr. ${ }^{882}$ Der Deursche Anwalt ist dem öffentlichen Interesse verpflichtet, den jüdischen Anwalt treibr sein (bei Weißler nicht näher bczeichneter) Charakter zum Verrat am Gemeinwolıl. Der Kompromiß zwischen „Staars-anwalt und jüdischem Advokat ist der ndeursche Rechtsanwalco. ${ }^{69}$

In dieser Tradition haben dann auch in der Weimarer Zeir eine Rcihe von mdeurschen Anwälten « ihre Auffassungen dargelegt. Richtig entfalten konnce sich diese deutschrumelnde Tendenz, denen die großen Vorbilder der freien Advokatur in England, Frankreich und den USA ebenso unbekannt waren wic dic theoretischen Grundlagen des bürgerlichen Rechtsstaates, erst in der Nazi-Zeit. ${ }^{70}$

Mit den jüdischen Anwälın hat die freie Advokatur in Deurschland ibr Rückgrat verloren. Max Alsberg beendete 1933 im Schrveizer Exil sein Leben. Wie seine Frau berichtete, war für ihn das Leben nur als deutscher Strafverteidiger denkbar. Deshalb schlug cr auch die ithm vom französischen Ministerpräsidenten angebotene Professur an der Sorbonne aus. Juljus Magnus starb im Konzentrationslager Theresienstadt, Adolf Friedlacnder kam seiner Deportation im Jahre 1942 durch den Freitod zuvor. Harry Litten, seit 1933 in Konzentrationslagern gefangen gehalten, wurde $1938 \mathrm{zu}$ Tode gefolcer. Hugo Sinzheimer, der sich nach dem Einmarsch der Deurschen in Amsterdam bis 1945 in ciner Dachkammer versteckr hielt, starb noch im selben Jahr an Entkräftung. Hachenburg, Neumann und Fraenkel gingen ins Exil. Die Liste umfaßte mehr als 4500 Namen, wenn ihre Geschichte geschrieben würde."

Mit den jüdischen Juriscen starb und emigrierte auch die advokatorische Rechoswissenschaft und die Tradition der freien Advokatur. In der noch 1933 angeferrigten und im Ausland neu aufgelegten Festschrift für Marrin Drucker hatren sie noch einmal ein letzres Zeugnis ihrer Bedeutung für cin demokrarisches Deutschland abgelegt. Damals ist ihre Stimme verstummt. Die verbliebenen \&deutschen « Rechts * anwälte von Dix ${ }^{\text {z2 }}$ über Grimms bis Noack, Raeke und Droege, aber auch die

68 Die Rechesanwaltschaft, a. 3. O., so Jahre freie Advokatur in Dcuzschland.

68 . Weißler, Geschichic der Rechisanwaltschaft, 1967 (Nachdruck 1909), S. 603.

69 Besonders deualich in WeiBler/Zeleer, Dic Rechisanwalischaft am Scheideweg, JW 11, 474ff. 0. 5621 If.

70 Vgl. z. B. die historische Ableizung bei Neubert, Anwaleschalt und Jusizverwaltung in Preußen in den leaten beiden Jahrbunderten, in: 200 Jahre Dienst an Reche, ( $\mathrm{Hg}$. Reichsjustizminister Gurner), 1936 S. 29 if If.

I Vgl. Goppinger, Der National sozialismus und die jüdischen Juristen, s963: Osder, a. a. O.; Heinrich, 100 Jahre Rechesanwaleskammer, a. a. O.

$72 \mathrm{Vgl}$. das positive Uneil von Osuler über Dix in: Die Deuschen Rechesanwalle, a. a. O., S. 224

73 Dazu Kirchheimer, Politische Justiz, S. ;68 I. 
Unzahl der stummen Angepaßıen repräsencierten eine andere deutsche Tradicion, dic man damals als wdeursch $\alpha$, vorher als svarerländisch « und fälschlich als dem Aligemeinwohl verpflichter, auf die Prokuratoren der Preußenkonige zurückführte. Sie wurden nach 1945 verstärkt durch die Vielzahl chemaliger NS-Beamter, Richter und Staatsanwälte selbst des Volksgerichtshofs, die von dem nunmehr freien Zugang zur Anwaltschaft profitieren konnten. Viele mögen sich geändert haben. Den Beitrag der jüdischen Advokaten zum Aufbau der freien Advokatur in Deurschland konnten sie jedenfalls nichs erseczen.

Die in letzter Zeit wieder verstärkt in Erscheinung tretenden Bemühungen der Anwalrsvereine um die freie Advokatur insbesondere im Bereich der Strafverteidigung sind fast 40 Jahre nach Kriegsende ein hoffnungsvolles Zeichen. Die Forschung zur Geschichte der freien Advokatur und ihrer Zerstörung in Deutschland kann dabei wichtige Hilfestellung geben, um positive Anknüpfungspunkte in der Anwaltstradition zu geben und vergessene Fortschritte und Ausarbeitungen nuizen zu können. Dies setž jedoch voraus, daß nicht die Geschichten deurscher Anwälte, sondern die Geschichte der freien Advokatur in Deutschland in Mittelpunkr der Betrachtung steht, die, und soviel kann mit Sicherheir gesagt werden, eng mit der Geschichte der jüdischen Advokaten in Deutschland verknüpft ist.

Suche zur Zusammerarbeit in meiner Kanzlei

\section{Anwaltskollegin oder Kollegen}

Die Kanzlei befindet sich in Kleinstadt mit bäuerlichem Umland (nordwestliches Mirtelf ranken). Es handelt sich um Allgemeinpraxis mit Schwerpunkt im Zivilrecht.

Hinsichtlich der Entwicklung der Zusammenarbeit stelle ich mir folgendes vor: Zunächst Zusammenarbeit in Form freier Mitarbeit. Bei guter Entwicklung der Zusammenarbeit (persönlich und in bezug auf den Umsatz) bin ich an Gründung eines Sozicıât gegen entsprechende finanzielle Beteiligung interessiert.

Der derzeitige Umsatz ist für den Einstieg einer Kollegin bzw. eines Kollegen ausreichend, müßte jedoch nach und nach noch gesteigert werden.

Der Grund, warum ich eine Sozietät anstrebe, liegt zum einen in der Verminderung des Risikos (z.B. durch Krankheit oder anderweitig bedingten Ausfall), zum anderen darin, daß ich meine Arbeitszeit verringern möchte.

Zuschriften unter Chiffre KJ/41284 an den Verlag. 